\title{
PROPUESTA METODOLÓGICA PARA EVALUAR MODELOS ACADÉMICOS EN INSTITUCIONES EDUCATIVAS
}

\author{
METHODOLOGICAL PROPOSAL \\ TO EVALUATE ACADEMIC MODELS \\ IN EDUCATIONAL INSTITUTIONS
}

\author{
Carlos Antonio Quintero Macías ${ }^{1}$ \\ https://orcid.org/0000-0003-4126-3790 \\ Carmen Silvia Peña Vargas ${ }^{2}$ \\ https://orcid.org/0000-0002-3537-6265
}

Recibido: septiembre 22, 2020 - Aceptado: octubre 13, 2020

\section{RESUMEN}

El presente artículo tiene como objetivo presentar una propuesta metodológica para evaluar modelos académicos, así como dar a conocer los resultados generales de su implementación en una institución educativa. Para su elaboración se analizaron modelos de evaluación educativos y académicos, así como la revisión de documentos institucionales y datos de entrevistas y focus group, a directivos y docentes. El aporte principal del artículo es la propuesta de evaluación, misma que puede utilizarse para analizar modelos educativos y académicos de otras instituciones educativas, preferentemente de nivel superior. En la parte final se muestran los resultados de la aplicación empírica de la metodología en un centro universitario, a través de la cual se analizó la estructura teórica y conceptual del modelo, su implementación real con distintos actores, así como los resultados a lo largo de los años, vistos a través de los impactos de sus egresados e indicadores institucionales. De tal forma que la propuesta metodológica puede aplicarse para analizar uno o todos los aspectos educativos que se presenten en las instituciones.

Palabras clave: modelo académico, modelo educativo, evaluación, instituciones educativas.

1 Doctorado en Educación, Universidad de Colima. Profesor de tiempo completo, Centro Universitario de los Valles, Universidad de Guadalajara. Miembro del Sistema Nacional de Investigadores. carlos.quintero@academicos.udg.mx

2 Doctorado en Educación, Instituto Tecnológico de Estudios Superiores de Occidente. Profesora de tiempo completo, Facultad de Ciencias de la Educación, Universidad de Colima. csilvia@ucol.mx 


\section{ABSTRACT}

The present article aims to present a methodological proposal to evaluate academic models, as well as to present the general results of its implementation in an educational institution. For its preparation, educational and academic evaluation models were analyzed, as well as the review of institutional documents and the application of interviews and focus groups to managers and teachers. The main contribution of the article is the evaluation proposal, which can be used to analyze educational and academic models of other educational institutions, preferably of a higher level. The final part shows the results of the empirical application of the methodology in a university center, through which the theoretical and conceptual structure of the model was analyzed, its real implementation with different actors, as well as the results that have been obtained over the years, seen through the impacts of its graduates and institutional indicators. In such a way that the methodological proposal can be applied to analyze one or all educational aspects that are presented in the institutions.

Keywords: Academic Model, Educational Model, Evaluation, Educational Institutions.

\section{INTRODUCCIÓN}

El presente artículo es el resultado de una investigación aplicada de largo alcance, la cual tuvo como propósito evaluar el modelo académico del Centro Universitario de los Valles (CUValles), de la Universidad de Guadalajara (UdeG). Para tal efecto, se revisaron modelos de evaluación educativa, así como instrumentos y metodologías para evaluar modelos educativos y académicos.

Con base en estos elementos, se estructuró una propuesta particular de evaluación, aplicada de julio de 2019 a febrero de 2020, con resultados positivos en relación con el cumplimiento de los objetivos planteados. La propuesta amalgama no solo los elementos teórico-conceptuales sino, sobre todo, las experiencias prácticas que se tuvieron en su aplicación.

El principal objetivo del artículo es dar a conocer la propuesta metodológica, esperando sirva de estructura y guía para otras instituciones educativas. De igual forma, se presentan de manera general algunos resultados logrados con su implementación.

Toda metodología que intente evaluar modelos educativos y académicos debe ser totalmente flexible, permitiendo que los actores involucrados la adecuen a sus necesidades particulares; así como también la redefinan en el trayecto, siendo este uno de los aportes de la propuesta metodológica de este artículo.

La Universidad de Guadalajara cuenta con un modelo educativo denominado «Siglo 21», el cual establece los principios y las máximas en torno a la educación de la institución. Es importante destacar que este modelo no solo implica la función sustantiva de docencia (mediación educativa), sino también la investigación y extensión universitaria, con una perspectiva de innovación e internacionalización; de tal forma que la universidad visualiza al proceso educativo como un todo integral que impacta en el desarrollo social de manera considerable. 
El modelo educativo Siglo 21 es un paradigma en que la Universidad de Guadalajara se sustenta, «es una fuerza orientadora del ser y del quehacer universitario; guía para desarrollar una cultura académica» (Universidad de Guadalajara, 2007: 17). Si bien propone una guía para desarrollar un estilo y una cultura para la universidad entera, también permite cierta flexibilidad para todos los actores y centros universitarios que componen a la Red Universitaria, de tal forma que los centros incorporan particularidades educativas sustentadas en sus contextos socioculturales.

Por su parte, el Centro Universitario de los Valles, al formar parte de la propia Universidad de Guadalajara, observa y se conduce con base en el modelo educativo mencionado, pero añade un modelo académico-pedagógico con características únicas y particulares, mismas que fueron evaluadas con la propuesta que a continuación se presenta.

\section{METODOLOGÍA}

Para realizar el presente artículo se realizó una investigación aplicada en el Centro Universitario de los Valles, consistente en evaluar su modelo académico. Se inició con la revisión de literatura en torno a la evaluación educativa, así como documentos sobre cómo realizar análisis de modelos educativos y académicos.

El estudio partió de la revisión de los modelos de Stufflebeam y Scriven; posteriormente, al analizar diversas propuestas evaluativas, la metodología fue reestructurándose hasta llegar a una con características únicas. También se utilizaron fuentes secundarias de información, sobre todo, investigaciones acerca del modelo CUValles y sobre el propio centro universitario, cuidando la objetividad de dicha información. De tal forma que el estudio abarcó una temporalidad aproximada de 19 años, ya que se tomaron documentos desde la creación del Centro en el año 2000, hasta el año 2019.

Cabe mencionar que cada medio de verificación utilizado tuvo un período diferente de años, dependiendo de los datos con los que se contaba, aspecto que se tomó en cuenta para la redacción de resultados. Es importante aclarar que al estar trabajando el Centro Universitario con un mismo modelo desde el año 2000, los resultados e impactos aplican para todos los estudios realizados y para todos los actores que en él participen.

La implementación de la propuesta en el CUValles rescató la opinión de egresados y empleadores, a través de estudios previamente elaborados; pero, sobre todo, se incluyó la revisión de documentos oficiales del Centro, en donde se encuentra plasmado el modelo académico.

Los instrumentos de recolección de información directa empleados, fueron entrevistas semiestructuradas realizadas a los directivos del Centro; así como focus group a coordinadores de carrera y docentes. Participaron directores de División, jefes de Departamento, doce coordinadores, y cincuenta y cuatro profesores de seis Departamentos.

El número de profesores entrevistados fue seleccionado con lo que en investigación cualitativa se conoce como punto de saturación, relacionado con el hecho de que todos los entrevistados manifestaron información similar, y por más profesores que se hubieran entrevistado, los resultados no cambiarían considerablemente. Cuñat (2007) manifiesta que «la saturación teórica se logra recogiendo información hasta que ya no surgen nuevas evidencias o sucesos que puedan informar del desarrollo del proceso. No hay una norma clara 
de corte, pero es importante saturar la información obtenida» (p. 5). En el mismo sentido, Blasco y Otero (2008) comentan que «se dejará de hacer entrevistas cuando se ha llegado al punto de saturación de la información, es decir el punto en el cual realizar entrevistas adicionales no aporta nada nuevo a la información que ya se tiene» (s.p.).

Finalmente, se analizaron datos proporcionados por la Coordinación de Control Escolar, relacionados con índices de egreso, titulación, ingreso, deserción, entre otros.

Del resultado final de la evaluación realizada, surge la propuesta metodológica que se presenta en este artículo, para evaluar modelos académicos.

\section{SUSTENTO TEÓRICO}

En relación con los modelos educativos, académicos y pedagógicos existe bastante literatura; no obstante, no hay un consenso en torno a qué se refiere con cada conceptualización, en parte porque el propio campo educativo y pedagógico así funciona, y en sí, todas las humanidades y ciencias sociales. De tal forma que las instituciones educativas deben tomar postura respecto a qué entienden por cada concepto y con base en ello estructurar sus procesos de trabajo.

\section{¿MODALIDAD O MODELO?}

La literatura en torno a las modalidades educativas así como a los propios modelos es ambigua; generalmente se describen como sinónimos o se definen utilizando otros términos como pedagógico o didáctico.

En México, la Secretaría de Educación Pública establece tres modalidades educativas: escolarizada, no escolarizada y mixta, con base en el Acuerdo 243 de 1998 (Diario Oficial, 1988: 1). De tal forma que una modalidad define particularmente las formas de trabajo, en relación con los elementos sincrónicos y asincrónicos de los actores, ya sea dentro de las instituciones educativas o a través de plataformas tecnológicas.

Por su parte, los modelos educativos y académicos definen los elementos organizacionales de las instituciones, y guardan estrecha relación con las modalidades de trabajo implementadas. Estos, estructuran y guían la implementación curricular de las carreras, por lo cual es importante que se precisen con claridad. En este sentido, un modelo educativo o académico define las formas de trabajo concretas y las particularidades que tendrá el acto educativo. Cuando se estructura un modelo generalmente se piensa en cierta modalidad, aunque los modelos pueden adaptarse según las circunstancias a distintas modalidades.

De esta forma, y debido a la pandemia por Covid 19, la mayoría de las instituciones educativas en México y en el mundo, migraron de una modalidad presencial a una mixta o a distancia, de tal manera que los modelos con los que trabajan debieron adaptarse (particularmente las funciones de docentes y estudiantes), con el apoyo de los procesos administrativos.

Un elemento central para las instituciones educativas es definir qué entienden por modelo y por modalidad y, sobre todo, los lineamientos con los que operarán, ya que su combinación orienta los procesos de trabajo. 
Por su conceptualización, los modelos educativos son estructuralmente más amplios que los modelos académicos, es decir, de los primeros se desprenden los segundos. Un modelo educativo generalmente estipula la razón de ser de la institución, su sentido social y, sobre todo, la misión, visión y los valores con que opera. En relación con el modelo educativo de la Universidad de Guadalajara, este propone «un proceso formativo centrado en el estudiante y sus modos de ser y aprender a ser, conocer, hacer, convivir, emprender y crear» (Universidad de Guadalajara, 2007: 8); ello marca un giro de $160^{\circ}$ a los modelos basados en el profesor y en la «transmisión» del conocimiento, enfatizando el rol activo que debe jugar el estudiante en el logro de sus aprendizajes.

El modelo educativo Siglo 21 de la UdeG, presenta las siguientes características: formar en la diferencia; entender al ser humano desde lo social; generar aprendizajes para la convivencia y propiciar el encuentro entre diferentes formas de pensar; reconocer al conocimiento como patrimonio de la humanidad; proporcionar una formación integral; y dar cabida a lo afectivo y pasional, además del conocimiento (Universidad de Guadalajara, 2007).

Dicho modelo guarda estrecha relación con la reforma universitaria de 1994, mediante la cual se crea la Red Universitaria de Jalisco, estableciéndose departamentos académicos en lugar de las escuelas y facultades tradicionales. Según Sánchez (1995): «La estructura departamental se caracteriza por el agrupamiento de profesores e investigadores en un departamento en torno a un campo especializado del conocimiento» (p. 3).

Por su parte, los modelos académicos refieren a la estructura operativa y organizacional de la institución, por supuesto en observancia directa de los modelos educativos. En el caso de la Universidad de Guadalajara, esta cuenta con un modelo académico «definido por la estructura orgánica de la institución, los procesos de gestión y administración con que dicha estructura da soporte al desarrollo de las funciones sustantivas» (Universidad de Guadalajara, 2007: 9). El modelo académico configura la operativización del modelo educativo y permite su óptimo desarrollo.

Entre sus características se encuentran las siguientes: está centrado en el estudiante; las formas de trabajo académico y administrativo tienen mayor flexibilidad; se presenta un modelo curricular semiflexible, y se estructura mediante un modelo departamental, en contraposición con los modelos napoleónicos de escuelas y facultades: el departamento académico disciplinar, multidisciplinar o por objeto de trabajo, es el núcleo donde se concentran los especialistas de una determinada disciplina o campo de estudio, para realizar tareas de investigación, de formación académica (docencia, tutoría, asesorías de proyectos) y de extensión (vinculación, difusión, promoción) (Universidad de Guadalajara, 2007: 36).

Con base en lo comentado, evaluar un modelo educativo o académico, implica considerar diversos elementos. La metodología que aquí se propone, puede adaptarse tanto a uno como a otro. Si bien se realizó pensando en modelos académicos, las instituciones que decidan implementarla pueden adaptar los elementos que aquí se mencionan, y aplicarla para dar cuenta de los procesos de trabajo, así como los impactos de estos.

Sánchez (1995) publicó un documento titulado «Modelos académicos predominantes en las instituciones de educación superior mexicanas: los currículos rígidos, semiflexibles y modulares», donde se vincula de manera directa al modelo académico con el currículum, asociando directamente la estructura organizacional con los procesos de enseñanza-aprendizaje. No obstante, como se ha visto, una institución de educación superior trabaja otros ejes o tiene más funciones sustantivas que solo la docencia. La misma Sánchez (1995) menciona que «los modelos académicos hacen referencia a la estructura académico-administrativa de la institución» (p. 2), a los procesos de trabajo que estructuran la operativización, sobre todo 
de las sesiones de aprendizaje. Comenta que la mayoría de las instituciones mexicanas se organizan por escuelas o facultades, y otras por departamentos académicos, cada una con características diferentes. Dentro de estas existen los documentos curriculares propios de las carreras, mismos que pueden tener características flexibles, semiflexibles, rígidas o modulares. En este caso se habla de procesos diferentes, por un lado, de modelos académicos y por el otro, de documentos curriculares.

Para este momento de la discusión, ya se han mencionado varios elementos que confluyen dentro de la estructura y operativización de una institución educativa: modalidades de trabajo, modelos educativos, modelos académicos y modelos curriculares, haciendo que el acto educativo sea complejo; por ello, no deben hacerse copias literales de procesos de trabajo exitosos en otras partes del mundo, ya que existe una gran cantidad de elementos a considerar.

\section{ESTUDIOS SOBRE MODELOS DE EVALUACIÓN}

Algunas propuestas para analizar modelos educativos y académicos, fueron revisadas para elaborar la que aquí se presenta. Son heterogéneas y abarcan un sinfín de elementos: desde los objetivos o fines de la evaluación, hasta los actores involucrados, pasando por valorar si se trata de una evaluación de trayecto o sumativa.

García (2010) menciona que debe distinguirse entre monitoreo y evaluación. El primero refiere a la implementación de los programas, sobre todo para que tengan relación con lo planeado; mientras que las evaluaciones son más completas y se definen como un examen más profundo. En este sentido, una evaluación debe considerar, además de la revisión propia del modelo, los impactos en los estudiantes y en la sociedad.

En relación con los fines de la evaluación, García (2010) comenta que se espera que las evaluaciones coadyuven en el aprendizaje de la organización, de forma que sirvan como elemento para la toma de decisiones, uno de los objetivos centrales de estas evaluaciones.

Otro elemento para las propuestas de evaluación, guarda relación con las fuentes de información y los propios indicadores, mismos que pueden ser cuantitativos y cualitativos. Generalmente la información cuantitativa es más fácil de obtener y de analizar (como son los resultados de exámenes de egreso, índices de titulación o deserción, porcentaje de estudiantes admitidos, entre otros). No obstante, los datos cualitativos son ricos, ya que «se espera que a través de ellos sea posible establecer inferencias acerca de la calidad de la enseñanza» (García, 2010).

Las inferencias ayudan cuando no existen estándares para realizar juicios comparativos, que sin duda son indispensables en la toma de decisiones. Por ejemplo: inferir si bajo determinada modalidad, el modelo en cuestión daría mejores resultados; lo cual es casi imposible de saber mediante datos cuantitativos, ya que en el proceso intervienen una gran cantidad de actores y procesos, de los cuales prácticamente no hay control. Por ello, un elemento interesante de la propuesta es que debe construirse durante el mismo proceso, partir de cuestiones objetivas dejando un margen de flexibilidad para que, sobre la macha, se hagan ajustes pertinentes al trabajo.

En 2016, la Universidad Autónoma Metropolitana-Cuajimalpa, realizó un proceso de evaluación de su modelo educativo, encontrando que hay una gran cantidad de variables a 
considerar en las evaluaciones: «rol del alumno, rol del docente, la visión de la institución, la estructura curricular y extracurricular, la oferta educativa» (Moreno, Espinoza, Solano y Fresán, 2016: 30), entre otros.

Para la realización de este estudio se conformó una comisión integrada por varias personas, mostrando que las evaluaciones de modelos educativos y académicos, por su complejidad, requieren de un equipo de trabajo, siendo muy difícil que uno o dos actores por sí solos puedan realizarlas.

Los actores que participaron en la evaluación referida coincidieron que «desde la génesis del proyecto se partió con una perspectiva de la evaluación como un proceso en construcción conjunta, donde cuentan todos los puntos de vista de los participantes» (Moreno et al., 2016: 32). De esta forma, el modelo utilizado consistió en una evaluación participativa, elemento que ayuda para que sea flexible.

Se parte de ciertos elementos concretos -como los objetivos de la evaluación y el equipo que trabajará en ella- y sobre la marcha se reestructurarán permanentemente con base en las circunstancias y los elementos que se vayan encontrando.

Un elemento de este estudio tiene que ver con la diferenciación entre la implementación de los modelos y lo que se declara en los documentos oficiales, ya que son dos cosas distintas. Podría hacerse la analogía con los currículums oficiales y ocultos, donde estos últimos terminan reconfigurando el escenario educativo. «Evaluar la implementación de un modelo educativo es una tarea imprescindible, porque solo a través de esta valoración podremos conocer la efectividad de la propuesta e identificar la distancia que existe entre lo que se pretendía y lo alcanzado» (Moreno et al., 2016: 34).

Por su parte, Gómez, López, Romo y de la Torre (2012), presentaron una evaluación que realizó la Universidad Autónoma de Chihuahua, donde se valoró el impacto de su modelo educativo. Las evaluaciones de impacto son importantes porque hacen referencia a la parte final del proceso; pueden dividirse en analizar los resultados educativos en cuestión de indicadores, así como también analizar impactos que sobrepasan las fronteras de la institución, en este caso impactos sociales.

El estudio realizado por la Universidad Autónoma de Chihuahua fue extenso, ya que abarcó a «1,953 encuestados de un total de 16 PE de nivel licenciatura» (Gómez et al., 2012: 108), a través de una metodología no experimental, sin control o manipulación del entorno. Este tipo de estudios corrobora lo mencionado anteriormente, en relación con el equipo de trabajo indispensable para realizar las evaluaciones de los modelos.

Por otra parte, de acuerdo con los modelos de evaluación educativa en general, se revisaron dos propuestas principalmente. La primera fue la metodología de Stufflebeam, desarrollada a partir de la década de los 70, conocida como modelo CIPP por sus siglas en inglés: Context, Input, Process y Product. Este modelo puede aplicarse prácticamente a todo lo que se desee evaluar: desde documentos curriculares, programas de estudio, incluso programas de intervención social. El modelo de Stufflebeam proporciona la siguiente información: necesidades que existen y hasta qué punto los objetivos propuestos las satisfacen; descripción del programa de intervención y análisis conceptual de la adecuación de la propuesta elegida a los objetivos; grado de realización del plan de intervención propuesto y descripción de las modificaciones; resultados obtenidos y grado en que se han satisfecho las necesidades (Bausela, 2003: 371). 
Un aspecto importante de este modelo evaluativo tiene que ver con el contexto de la institución educativa, mismo que determina la operativización de los propios modelos. «Consiste en identificar las virtudes y defectos de algún objeto, como una institución, un programa, una población escogida o una persona, y proporcionar una guía para su perfeccionamiento. Es decir, trata de identificar las características del entorno en el cual el programa se va a llevar a cabo» (Bausela, 2003: 367). Un buen análisis contextual per se, puede dar cuenta de los cambios que deben realizarse a los modelos educativos o académicos, ya que su éxito depende sobremanera del lugar donde se aplica y las personas que lo circunscriben y trabajan. Cabe mencionar que los contextos guardan relación no solo con los aspectos educativos, sino también socioculturales.

Por su parte, las evaluaciones de entrada son «utilizadas para determinar cómo utilizar los recursos disponibles para satisfacer las metas y los objetivos del programa. La principal orientación de una evaluación de entrada es ayudar a prescribir un programa mediante el cual se efectúen los cambios necesarios» (Bausela, 2003: 368).

Todo modelo se modifica y reestructura constantemente cuando es puesto en práctica, con base en múltiples realidades y contingencias, de forma que al analizarlos es importante no solo revisar su estructura, sino también sus procesos de implementación. Este tipo de evaluación es conocida como evaluación del proceso: «es una comprobación continua de la realización de un plan. Persigue proporcionar la información necesaria para determinar si el programa llevado a cabo sería aceptado tal cual, o corregir aquellos aspectos que no han funcionado» (Bausela, 2003: 369).

Asimismo, existen algunos elementos que evidencian los impactos de la educación en sus estudiantes, en sus egresados, así como en la sociedad en general. Este tipo de evaluación se conoce como evaluación del producto, con base en el modelo de Stufflebeam, el cual tiene el objetivo de «valorar, interpretar y juzgar los logros de un programa [...]. Con el fin de tomar decisiones de reciclaje» (Bausela, 2003: 370). La idea del reciclaje en educación implica rehacer, reorientar o reutilizar los elementos pedagógicos y administrativos con el fin de mejorar los procesos.

Otro de los modelos clásicos de evaluación educativa es el de «Evaluación sin metas», de Scriven: «la evaluación debe ser un examen crítico de la institución, proyecto, programa, proceso u objeto, sin tomar en cuenta sus objetivos planteados» (Ruiz, 2016: 34). Este tipo de evaluación se enfoca en valorar los resultados y efectos que produce el programa, o en este caso, el modelo académico. Se utiliza sobre todo para realizar estudios de egresados de manera cualitativa. Cabe mencionar que los egresados tienen un impacto mayor que implica no solo los mercados laborales; por ello será importante analizar el impacto en sus familias y en la sociedad.

Con el modelo de Scriven se valoran también las contingencias educativas, las cuales se presentan sobre todo en nivel superior, al tratarse de instituciones heterogéneas, y más en la Universidad de Guadalajara, debido al número de estudiantes y docentes con los que cuenta.

El modelo de evaluación de Scriven se complementa con el de Tayler, denominado «De objetivos conductuales». Los objetivos propios de los programas educativos e instituciones también deben ser tomados en cuenta por las evaluaciones, a fin de determinar el grado de alcance en su implementación. Con este modelo se contrasta la información obtenida con las metas que en un inicio tiene la institución educativa, en relación con los resultados propios de la educación; son útiles al evaluar indicadores cuantitativos (como índices de deserción, egreso, titulación, aprovechamiento, entre otros). 
En relación con los modelos de evaluación -sobre todo de corte cuantitativo- es importante que estos tomen en cuenta los indicadores o las categorías de indicadores, de manera que se conviertan en una guía al momento de realizar las evaluaciones, ganando así objetividad.

Al respecto, Corvalán (2000) recuperó una serie de indicadores planteados por la Oficina Regional de Educación para América Latina y el Caribe, para realizar un «Modelo conceptual de indicadores en Educación», estos fueron seleccionados con base en los siguientes aspectos: «Contexto, recursos, alcance de la educación, funcionamiento de la educación, equidad en las oportunidades, calidad de la educación, e impacto social» (Corvalán, 2000: 150). Si se observa, dichos indicadores guardan relación directa con el modelo propuesto por Stufflebeam para realizar evaluaciones educativas.

\section{ANÁLISIS Y DISCUSIÓN DE LOS RESULTADOS}

El presente apartado se divide en dos para una mejor lectura: la propuesta metodológica para evaluar modelos académicos, y los resultados que se tuvieron al aplicar de manera empírica dicha propuesta en un centro universitario.

\section{Propuesta metodológica para evaluar modelos académicos}

Este sub-capítulo es el de mayor trascendencia en todo el artículo, si bien interesa conocer los resultados propios de la implementación, el objetivo central es dar a conocer una propuesta de evaluación que sirva para implementarse en otras instituciones.

Primeramente, debe mencionarse que la evaluación de un modelo educativo -académico o pedagógico- de una institución de educación superior, contempla sus funciones sustantivas, mismas que generalmente hacen referencia a la docencia o mediación educativa, a la investigación, y a la vinculación y extensión. Si no se observan estos elementos como un todo, es importante que se mencione al inicio de la evaluación, de tal forma que esta se vaya delimitando.

Como se ha comentado, nuestra propuesta metodológica se enfoca principalmente en el eje de docencia o mediación educativa, aunque se analizan también, en menor medida, algunos elementos de los otros ejes.

Dicha propuesta se denominó, en un primer momento, «Evaluación participativa en construcción», pues involucró a estudiantes, académicos, directivos y administrativos del centro universitario, quienes además de proporcionar información, fueron reorientando el rumbo de esta. Una vez que se inició con las actividades, fue necesario definir un comité o grupo de trabajo para estructurar el modelo y realizar la evaluación. Dicho comité se conformó por seis personas de distintas profesiones, lo que permitió una pluralidad de ideas, enriqueciéndolo.

Otro paso inicial fue clarificar y precisar los objetivos de la evaluación ya que, como se señaló, la educación abarca muchos elementos; por ello, si los evaluadores no precisan y delimitan los alcances de su intervención, pueden perderse con facilidad. Los objetivos quedaron establecidos de la siguiente manera: 
1. Analizar la estructura oficial del modelo en los documentos oficiales.

2. Examinar la implementación del modelo en el centro universitario.

3. Estudiar los impactos del modelo: resultados educativos y sociales.

Si se observa, cada uno de estos objetivos es un trabajo de evaluación per se, lo cual habla de la amplitud del trabajo empírico realizado, pero también de todos los elementos que pueden abarcarse con la metodología de evaluación.

La propuesta, como indicamos, se sustenta particularmente en el modelo de Stufflebeam, ya que presenta de manera clara los pasos a seguir para aplicar las evaluaciones. Con base en este modelo se desarrollaron cuatro categorías de análisis, cada una con sus respectivos medios de verificación.

a) Contexto demográfico, sociocultural y económico del CUValles.

- Comparar orígenes y antecedentes con los que nace CUValles, con los elementos socioeconómicos y culturales actuales.

b) Modelo CUValles en los documentos oficiales, acceso a la educación y recursos materiales.

- Análisis de la infraestructura tecnológica con la que cuenta el Centro para relacionarla con los documentos oficiales de creación y documentos oficiales institucionales.

- Observar las características de infraestructura del CUValles y examinar la opinión de los responsables de esta área.

- Considerar los puntajes de ingreso de los estudiantes en el College Board y las calificaciones con que ingresan.

- Analizar si los programas Talleres de formación integral, Tutorías, Lenguas extranjeras, Salud integral, y Habilidades cognitivas ${ }^{3}$, se encuentran en los documentos de creación del CUValles y en algunos documentos oficiales institucionales.

- Examinar el número de elementos teóricos -desarrollados o referenciados- que se encuentran en los documentos oficiales de creación y cómo son descritos, y compararlos con el número total de elementos teóricos que se incluyen en los documentos oficiales de creación del centro.

- Revisar el número de elementos teóricos no desarrollados ni referenciados.

- Comparar la estructura formal del modelo CUValles con el modelo educativo Siglo 21 de la UdeG.

- Relacionar las herramientas tecnológicas que utilizan los docentes, con lo establecido en los documentos oficiales de creación.

3 Estos programas forman parte de una estructura académico-administrativa para fortalecer los aprendizajes de los estudiantes, con el objetivo de que desarrollen una formación integral. Cada institución que aplique la metodología, valorará sus propios programas y estrategias para incluirlos en la evaluación. 
- Analizar la capacitación que reciben los nuevos profesores en relación con el modelo del Centro.

- Examinar la planta docente y estudiantil actual, y compararla con lo establecido en los documentos oficiales de creación.

c) Procesos educativos e implementación del modelo académico.

- Relacionar la estructura de los cursos en plataforma tecnológica, con lo establecido en los documentos oficiales de creación del CUValles o en documentos oficiales institucionales.

- Examinar el uso «real» que los docentes y estudiantes le dan a la plataforma tecnológica, y relacionarlo con el «deber ser».

- Analizar la forma en que los docentes trabajan presencialmente sus cursos, y relacionarlo con lo establecido en los documentos oficiales.

- Estudiar los tipos de actividades extra-aula que realizan los docentes y la forma de evaluar estas actividades, así como los tiempos en que asignan calificaciones y la retroalimentación a los estudiantes, y compararlo con lo establecido en los documentos oficiales.

- Relacionar la capacitación y formación recibida de los profesores, con la manera en que trabajan sus cursos.

- Analizar cómo trabajan el proceso de aprendizaje los estudiantes, dentro y fuera de las aulas.

- Examinar las interpretaciones que hacen estudiantes, docentes y administrativos de los conceptos sobre el modelo CUValles.

- Comparar los elementos actuales -en cuestiones pedagógicas- con los elementos pedagógicos que se encuentran en los documentos oficiales.

d) Resultados académicos e impacto social.

- Porcentaje de alumnos que obtienen una calificación aprobatoria en el EGEL Ceneval, y compararlos con otros centros universitarios y con indicadores nacionales.

- Porcentaje de egreso, eficiencia terminal y deserción del CUValles, y compararlos con indicadores nacionales y con otros centros universitarios de la UdeG.

- Número y porcentaje de estudiantes titulados, tiempos de logro y sus modalidades ${ }^{4}, \mathrm{y}$ compararlos con indicadores nacionales y otros centros universitarios de la UdeG.

- Porcentaje de inserción laboral de los egresados y tiempos de inserción, y compararlos con indicadores nacionales y otros centros universitarios de la UdeG.

4 Es importante analizar en cuánto tiempo se titularon después de haber egresado, así como las formas, ya que ello dice mucho de la estructura académica de la institución, sobre todo en relación con productos para la titulación (como las tesis o tesinas). 
- Comparar el rendimiento académico de los estudiantes con la percepción que estudiantes y maestros tienen de este indicador.

- Número de recomendaciones emitidas por organismos acreditadores, nacionales e internacionales ${ }^{5}$.

- Número de estudiantes atendidos y percepción (utilidad para los estudiantes y para las carreras, resultados, elementos positivos y negativos) de los programas: Talleres de formación integral, Tutorías, Lenguas extranjeras, Salud integral, y Habilidades cognitivas del CUValles, que poseen estudiantes, profesores, administrativos y directivos. Observar si estos programas se encuentran en los documentos de creación del CUValles y en algunos documentos oficiales institucionales.

- Comparar las calificaciones de ingreso de los estudiantes con las calificaciones de egreso.

Como se ha mencionado, además del modelo de Stufflebeam, se implementaron otros dos modelos particulares: el enfoque de objetivos conductuales y el enfoque que prescinde de objetivos. Si bien estos modelos parecen opuestos, al momento de su aplicación se complementan para analizar distintos elementos de manera cualitativa y cuantitativa. El primero -enfoque de objetivos conductuales- analiza las metas establecidas tal como están formuladas y recaba pruebas para comprobar si se han alcanzado (House, 2000: 28). Este modelo evaluativo fue propuesto por Tyler a mediados de los años cincuenta del siglo pasado. El segundo -el enfoque que prescinde de los objetivos- posee un carácter más cualitativo; en este, el evaluador debe investigar todos los resultados, muchos de los cuales son efectos colaterales imprevistos que pueden ser positivos o negativos (House, 2000: 30).

Este último modelo fue propuesto por Scriven en la década de los setenta también del siglo pasado, quien prestó atención a «desarrollar el concepto de necesidad como fundamento de la evaluación, por tanto, la evaluación independiente de los objetivos se basa en las necesidades del usuario y no de los objetivos de los productores» (House, 2000: 32).

De esta forma, la metodología a trabajar retoma tres modelos evaluativos: el modelo CIPP, el modelo de objetivos conductuales, y el modelo que prescinde de los objetivos. Así mismo, esta metodología propone utilizar el análisis del discurso, tanto con los documentos oficiales escritos, como con sus principales actores. Para esto último se utilizaron entrevistas a profundidad y focus group con profesores, siendo necesario realizar guiones de preguntas y elementos a trabajar.

Cabe mencionar que durante décadas, la idea predominante de la evaluación se relacionó directamente con «medir», dándole peso únicamente a dimensiones e indicadores cuantitativos. Actualmente, la evaluación de impacto es valorada como un proceso amplio y global, en el que al abordaje cuantitativo se agregan técnicas cualitativas (Gómez et al., 2012).

La propuesta aquí presentada, analiza los modelos académicos considerando los tres objetivos generales antes mencionados; para lo cual no basta con analizar los documentos oficiales donde se encuentra el modelo -ya que ello es solo una parte del «deber ser»-, sino va más allá y evalúa su implementación. Esto es trascendental para que los modelos operen con base en lo establecido, siendo uno de los elementos en los que debe ponerse más atención.

5 En este caso se refiere a las acreditaciones institucionales realizadas por organismos autónomos, a los cuales la COPAES (Consejo para la Acreditación de la Educación Superior) les otorga el reconocimiento para hacerlo. Estas acreditaciones ayudan para posicionar académicamente a la instrucción y gestionar recursos. COPAES: www.copaes.org 
Otro de los elementos centrales de la metodología es partir de documentos existentes de acuerdo con los objetivos del estudio, sobre todo aquellos realizados desde el tiempo en que el modelo evaluado está operando, de tal forma que sus resultados guardan una estrecha relación con este. Lo más importante de examinar estos estudios es ganar tiempo y ahorrar esfuerzo, ya que analizar toda la institución educativa y todos sus actores, es una tarea titánica y prácticamente imposible.

En relación con la propuesta aquí presentada, se enfatiza que esta debe redefinirse por los propios evaluadores, tomando en cuenta los elementos que crean pertinentes para cumplir los objetivos propuestos. También es importante considerar los instrumentos para recoger información. En este caso, al trabajar con docentes y coordinadores se utilizó la técnica focus group, con sesiones de grabaciones de dos horas cada una aproximadamente.

En esta ocasión, para la aplicación de la metodología no se trabajó con estudiantes; de haberlo hecho se utilizarían como instrumentos las encuestas y los cuestionarios cerrados, ya que permiten un análisis más rápido de la información. De hecho, uno de los estudios que se revisó, realizado por el CUValles en 2014, aplicó más de 1,500 encuestas a estudiantes, lo cual requirió un sistema complejo de análisis.

Un elemento a considerar para aplicar la metodología se relaciona con los procesos político-administrativos de la propia institución, tanto los que se encuentran en su normativa institucional, como aquellos ocultos que la impactan. Es fundamental saber que los procesos de evaluación, al arrojar información para establecer juicios y tomar decisiones, no siempre son bien vistos por todos los actores; si bien se espera que estos mejoren los procesos académicos, en muchas ocasiones reestructurarán a la propia institución, aspecto que puede no gustar o incomodar a ciertas personas.

De esta forma, el tacto político de los evaluadores será fundamental para el éxito de este proceso. Este elemento, en muchas ocasiones intangible, depende de aspectos como: una buena comunicación; saber llegar a acuerdos; trabajo en equipo; incluso saber cabildear las ideas con las personas que podrían complicar el proceso evaluativo. Esto es tan importante como la propia estructura objetiva de la evaluación (pudiendo entorpecerla o detenerla por completo).

En relación con los impactos de los modelos en el entramado sociolaboral, es esencial acudir con egresados y empleadores, quienes tienen información relevante -de primera mano- para cumplir con este objetivo. Para ello, se realizará un muestreo por carreras o por generaciones, dependiendo del alcance del estudio y del tamaño de la institución. Generalmente las instancias de Control escolar y de Egresados guardan información -como correos electrónicos y domicilios-, pero puede emplearse el método «bola de nieve» para localizar tanto a egresados como empleadores.

Una vez recuperada y sistematizada la información, se redactan los resultados. Es importante cuidar los objetivos de la evaluación, así como considerar los efectos colaterales que surgieron durante el proceso, haciendo un trabajo más interesante. Al trabajar con esta metodología se recabará bastante información que no ayuda para los fines establecidos, por lo que debe dejarse de lado en el documento final.

\section{Implementación empírica de la propuesta metodológica}

A continuación, se presentan algunos resultados obtenidos con la implementación de la metodología. Cabe señalar que la información recabada fue bastante, ya que se partió de tres 
grandes objetivos generales, pero para efectos del presente artículo se hace una presentación sucinta de los elementos más significativos.

Conocer el contexto demográfico, sociocultural y económico de los estudiantes que asisten al centro universitario y de los pobladores de la región, es fundamental para constituir una estructura organizacional, ya que de ello dependerá la calidad de la educación.

En un inicio, para establecer la forma de trabajo del CUValles, se tomaron en cuenta los siguientes elementos: dispersión geográfica de los estudiantes; economía de las familias; necesidad de emplearse a la vez que se estudia, de tal forma que los estudiantes y las propias familias pudieran solventar los gastos que esto implica.

En la comparativa de información, desde 2000 a la fecha, se observó que la población analfabeta disminuyó del $8 \%$ al $4.4 \%$. Sin embargo, la cifra sigue estando por encima del promedio estatal. Por su parte, los ingresos económicos y el empleo son dos elementos fundamentales para el bienestar de las personas, mismos que permiten acceder y permanecer en las escuelas. Por ello, contar con ingresos que permitan atender estas necesidades, debe ir siempre de la mano del fenómeno educativo. La información recabada evidencia que más del $70 \%$ de los habitantes se encuentran por debajo de la línea de bienestar actual; esto es preocupante ya que muestra la precariedad económica en la región, misma que afecta sin duda a los estudiantes del centro universitario (Instituto de Información, Estadística y Geografía, 2019a; CONEVAL, 2019).

Al vincular el dato anterior con la educación formal, se observa que difícilmente los niños y jóvenes puedan mantenerse en las escuelas y solventar los gastos que ello implica. Si se comparan los datos de la Región Valles con la Zona Metropolitana de Guadalajara, puede advertirse una marcada diferencia, ya que en esta última solo el $6.9 \%$ de la población se encuentra con un ingreso inferior a la línea de bienestar mínimo, mientras que el 35.3\% de la población cuenta con un ingreso inferior a la línea de bienestar (Instituto de Información Estadística y Geografía, 2019b).

En lo que compete al modelo CUValles en documentos oficiales, se encontró que este es un «tipo ideal», que como tal solo puede encontrarse en dichos documentos, ya que la realidad que presenta el Centro hace que el modelo se reconstruya con el paso del tiempo, acoplándose a las diversas circunstancias. De esta forma, los modelos educativos y académicos necesitan repensarse y actualizarse constantemente.

Es importante que los modelos sean entendidos no como una camisa de fuerza que limite el trabajo, sino como un elemento de apoyo y guía para todo el fenómeno educativo; de esta forma se coincide con Bourdieu (1997), en el sentido de observar los modelos como estructuras estructurantes de la realidad. Como afirma Arreola (2012), «el modelo es una construcción teórica que emplea la ciencia para describir una realidad, jerarquizada, funcional y operacional mediante la cual estudiar la realidad» (p. 93). De esta forma, el modelo CUValles funciona como un «tipo ideal».

Dos de los sustentos teóricos centrales del modelo son el constructivismo y el aprendizaje autogestivo; los estudiantes aprenden no solo competencias disciplinares propias de cada carrera, sino sobre todo elementos pedagógicos generales.

Un elemento encontrado por Arreola (2012), es que a los estudiantes de nuevo ingreso les cuesta trabajo acoplarse al modelo, ya que vienen precedidos de un modelo tradicional, donde el rol del docente y del propio estudiante es muy diferente al empleado en 
el CUValles. En este sentido, la formación académica y cultural de los jóvenes determina el proceso educativo de la institución y, por ende, los resultados que se obtendrán.

En lo que compete a los documentos oficiales del modelo, se encontró que una sola vez aparece el adjetivo mixto para definirlo; también se presentan conceptos como no convencional o presencial optimizado, este último es el que lo define oficialmente. Se hallaron también 52 elementos teórico-conceptuales.

En relación con la intencionalidad del modelo, en el documento «Propuesta de creación del CUValles» (2004), se menciona que «el problema educativo que se plantea resolver es el viejo y enigmático proceso del aprendizaje, y su relación con el tiempo y el espacio», es decir, operar con un modelo flexible que optimice los trabajos.

En lo que corresponde a la implementación del modelo, se analizó un estudio realizado por la consultora ACSI (2017), en el cual los egresados manifestaron en un 37\% que se encontraban muy satisfechos con su trabajo actual, y el 51\% estaban satisfechos. Estos datos evidencian que la formación recibida respondió a sus expectativas, y por supuesto, que influye en la obtención de los empleos y en las actividades que realizan.

El estudio menciona que el $54.3 \%$ de los profesores indicaron que no es necesario actualizar la modalidad presencial optimizada del Centro, ya que «no es el modelo el que está fallando, sino la falta de identificación con el modelo por parte de los docentes, el compromiso y la selección de docentes» (ACSI, 2017).

En las entrevistas realizadas a docentes, administrativos y directivos sobre la implementación del modelo, se encontró que estos actores conocen e identifican sus elementos. También se preguntó a los docentes si consideran que el modelo es el adecuado para la formación de los estudiantes, y qué elementos añadirían o eliminarían para fortalecerlo. Las respuestas fueron interesantes: consideran que el modelo es el adecuado para trabajar en el Centro, sobre todo por las características de la región y las tendencias a nivel internacional. No obstante, es indispensable modificar algunos elementos de su estructura y sobre todo de su implementación, como, por ejemplo: una mejor inducción y capacitación a los nuevos estudiantes y profesores; un adecuado seguimiento al trabajo docente, y sobre todo que los procesos académicos se vinculen de mejor forma con los administrativos.

En lo que compete a los impactos del modelo, se dividieron en dos categorías: resultados académicos e impacto social.

En la primera categoría se debe mencionar que el Centro tiene una tasa elevada de egreso, misma que al compararla con los titulados disminuye drásticamente. De igual forma, los resultados en los exámenes nacionales de egreso no son del todo positivos.

En lo concerniente al impacto social de la educación, se observó que es un elemento importante que permite analizar los resultados de la propia institución. En 2009, el CUValles realizó un estudio sobre trayectorias profesionales de sus egresados, encontrando elementos significativos que vale la pena rescatar: «la poca capacidad productiva que se expresa en una insuficiente estructura productiva en la Región, altos costos de producción y escasa diversificación de cultivos son un obstáculo para que la economía regional pueda absorber fuerza de trabajo con un alto nivel de capacitación» (Montiel, Castillo y Ayala, 2009: 95).

De tal forma que valorar a los egresados por la realización de actividades relacionadas exclusivamente con su formación disciplinar, no sería justo ni para ellos ni para la propia institución educativa. 
Un punto importante encontrado por el estudio referido, guarda relación con la utilidad que los propios egresados manifiestan de los conocimientos adquiridos en el Centro, al respecto comentaron que «el pensar creativamente y el desarrollarse de manera independiente, son las capacidades que mayormente adquirieron durante su proceso de aprendizaje en el CUValles» (Montiel et al., 2009: 101), esto guarda relación directa con el modelo académico que se trabaja en el Centro.

Asimismo, el estudio de la consultora ACSI (2017), encontró un dato relevante relacionado con los impactos educativos del CUValles: el 83\% de los egresados entrevistados manifiestan que trabajan, aspecto que habla de una inserción laboral alta.

\section{CONCLUSIONES}

Las evaluaciones de modelos educativos y académicos requieren delimitarse en algunos aspectos, ya que son múltiples los elementos que envuelven al fenómeno educativo en cualquier institución. Si bien, la propuesta metodológica presentada puede trabajarse con todos los aspectos educativos que se desee, es importante que los responsables de la evaluación precisen sus objetivos y, en dado caso, delimiten los aspectos que no serán considerados.

Es necesario que este tipo de evaluaciones se construyan de manera permanente, con base en un modelo de evaluación flexible, que oriente sobre ciertos elementos a tomar en cuenta al momento de evaluar, pero que en ningún momento sea excluyente de otros aspectos que pueden considerarse, y mucho menos que funcione como una «camisa de fuerza».

Lo más importante al realizar estas evaluaciones es analizar los propios objetivos de la institución, las necesidades de trabajar este tipo de estudios y, sobre todo, que el equipo de trabajo reelabore metodologías -como la que aquí se presenta-, adaptándolas a sus necesidades.

Un elemento a considerar en la reconstrucción de los modelos para realizar evaluaciones, son los recursos humanos con los que se cuenta, y los elementos político-administrativos en torno a la misma, factores que pueden obstaculizarla o facilitar que se avance más rápido.

La aplicación empírica de la metodología permitió cumplir con los tres objetivos establecidos en un inicio; así mismo, facilitó el incorporar distintos elementos que se fueron recabando en el trayecto, haciendo de esta una metodología flexible.

\section{REFERENCIAS}

ACSI (2017). Análisis pedagógico del Centro Universitario de los Valles. México: CORPORATIVOACSI.COM.

Arreola, C.M.I. (2012). Tesis doctoral «Evaluación holística del modelo pedagógico del Centro Universitario de los Valles de la Universidad de Guadalajara». Universidad de Valladolid, España. 
Bausela, H.E. (2003). «Metodología de la Investigación Evaluativa: Modelo CIPP». Revista Complutense de Educación, 14(2), 361-376. Recuperado el 18 de septiembre de 2019, de https: / / revistas.ucm.es/index.php/RCED/article/view / 17241

Becker, S.G. (1994). Human Capital: A Theoretical and Empirical Analysis with Special Reference to Education. 3rd Edition. USA: The University of Chicago Press.

Blasco, H.T. y Otero, G.L. (2008). «Técnicas cualitativas para la recogida de datos en investigación cualitativa: La entrevista (II)», Nure Investigación, (34). Recuperado el 11 de marzo de 2012, de https:/ / www.nureinvestigacion.es/OJS/index.php/nure/article/ view/379

Bourdieu, P. (1997). Razones prácticas sobre la teoría de la acción. París: Éditions du Seuil.

CONEVAL. (2019). Evolución de las líneas de pobreza por ingresos. Recuperado el 23 de octubre de 2019, de http:/ / www.coneval.org.mx/Medicion/MP/Paginas/Lineas-de-bienestar-y-canasta-basica.aspx

Corvalan, A.M. (2000). Desarrollo de indicadores en educación en América Latina y el Caribe. UNESCO. Recuperado el 12 de septiembre de 2019, de https://unesdoc.unesco.org/ ark:/ 48223/pf0000161769

Cuñat J. R. J. (2007). «Aplicación de la Teoría Fundamentada (Grouded Theory) al estudio del proceso de creación de empresas». Decisiones basadas en el conocimiento y en el papel social de la empresa: XX Congreso anual de A.E.D.E.M. 2. Recuperado el 11 de marzo de 2012, de http: / / dialnet.unirioja.es / servlet/ articulo?codigo=2499458

Diario Oficial (1998). Secretaría de Educación Pública. Recuperado el 23 de octubre de 2019, de https: / / www.aplicaciones.abogadogeneral.ipn.mx/ PDFS / REVOES / ACUERDO_243.pdf

García, C.B. (2010). «Modelos teóricos e indicadores de evaluación educativa». Revista Electrónica Sinéctica, (15), 1-21. Recuperado el 18 de noviembre de 2019, de https:// www.redalyc.org/pdf/998/99815165002.pdf

Garrido, T.C. (2007). «La educación desde la teoría del capital humano y el otro». Educere, 11(36), 73-80. Recuperado el 24 de julio de 2012, de http:/ / redalyc.uaemex.mx / redalyc/pdf/356/35617701010.pdf

Gómez, O.E., López, D.J.C., Romo, G.J.R. y de la Torre, A.A. (2012). Resultados de la evaluación de impacto del modelo educativo de la Universidad Autónoma de Chihuahua. Primer Congreso Internacional de Educación. Recuperado el 24 de septiembre de 2019, de http: / / cie.uach.mx/cd/docs/area_01/a1p8.pdf

House, E.R. (2000). Evaluación, ética y poder. Tercera edición. España: Morata.

Instituto de Información, Estadística y Geografía (2019a). Valles Diagnóstico de la Región. Recuperado el 13 de noviembre de 2019, de https:/ /iieg.gob.mx/ns / wp-content / uploads / 2019/07/10-Valles-Diagn\%C3\%B3stico.pdf

Instituto de Información, Estadística y Geografía. (2019b). Guadalajara Diagnóstico municipal. Recuperado el 13 de noviembre de 2019, de https://iieg.gob.mx/contenido/ Municipios/GDL2019.pdf

Moreno, O.T., Espinosa, M.M., Solano, M.E.E. y Fresán, O.M.M. (2016). «Evaluación de un Modelo Educativo Universitario: Una perspectiva desde los Actores». Revista Ibe- 
roamericana de Evaluación Educativa, 9(2). Recuperado el 12 de agosto de 2019, de https: / / revistas.uam.es/index.php/ riee/article/view / 6655

Montiel, A.I., Castillo, G.V.M., y Ayala, R.S. (2009). Mercados de trabajo e inserción laboral de los egresados del CUValles. México: Universidad de Guadalajara.

Quintero, M.C.A. (2014). Tesis doctoral «Los egresados del CUValles como agentes de desarrollo social. Un análisis desde el campo del trabajo y el campo familiar». Universidad de Colima, México.

Ruiz, L.E. (2016). Propuesta de un modelo de evaluación curricular para el nivel superior. Una orientación cualitativa. México: Universidad Nacional Autónoma de México.

Sánchez, S.M.D. (1995). «Modelos académicos predominantes en las instituciones de educación superior mexicanas: los currículo rígidos, semiflexible, flexible y modular», Modelos académicos. México: ANUIES.

Universidad de Guadalajara (2004). Propuesta de Creación del Centro Universitario de los Valles. México: Universidad de Guadalajara.

Universidad de Guadalajara (2007). Modelo educativo siglo 21. México: Universidad de Guadalajara. 